\title{
Estrategias gerenciales para fomentar las competencias laborales en el Sector Hotelero de la Costa Caribe Colombiana
}

\author{
García Guiliany, Jesús Enrique* \\ Durán, Sonia Ethel** \\ Hernández, Janeth Carolina*** \\ Moreno, María Elena**** \\ * Universidad Simón Bolívar, Barranquilla, Colombia \\ **Fundación Universitaria Colombiana UNICOLOMBO, \\ Cartagena, Colombia \\ *** Universidad Privada “Dr. Rafael Belloso Chacín” URBE, \\ Maracaibo, Venezuela \\ ****Universidad del Zulia, Maracaibo, Venezuela \\ Correo autor principal: jesus.garcia@unisimonbolivar.edu.co
}

\begin{abstract}
Resumen
El estudio se orientó a identificar las estrategias gerenciales en el desarrollo de competencias laborales para crear ventaja competitiva en Pymes de Barranquilla Atlántico. El proceso metodológico aplicado, fue descriptivo, con un diseño no experimental de campo. La población estuvo conformada por treinta nueve (39) empleados administrativos de empresas constructoras, ubicadas en el distrito Barranquilla. Para recolectar los datos se utilizó un cuestionario constituido de 67 items, con alternativas tipo escala likert. Para el cálculo de la confiabilidad se utilizó el método de alfa Cronbach, donde se obtuvo un valor de 0.84 , determinando que el instrumento era altamente confiable. Los datos obtenidos fueron tabulados de acuerdo a los reactivos e interpretados estadísticamente mediante frecuencia absoluta y relativa, calculando los puntajes de tendencia central y desviación estándar, representados por tablas. Los resultados indican que las estrategias gerenciales aplicadas, establece la secuencia coherente de las acciones a realizar, por lo cual están orientadas al desarrollo de las competencias de los empleados, en virtud de alinearse a las necesidades y procesos que desde el entorno surgen para navegar en la competitividad de estas organizaciones. Estableciendo que una estrategia adecuadamente formulada, lleva consigo el alcance de objetivos establecidos.
\end{abstract}

Palabras clave: Estrategias gerenciales, competencias, competitividad, necesidades, procesos 


\title{
Management strategies to promote labor competencies in the Hotel Sector of the Colombian Caribbean Coast
}

\begin{abstract}
The study was oriented to identify managerial strategies in the development of labor competencies to create competitive advantage in Pyrenees of Barranquilla Atlantico. The applied methodological process was descriptive, with a non experimental field design. The population consisted of thirty nine (39) administrative employees of construction companies, located in the Barranquilla district. To collect the data, a questionnaire consisting of 67 items was used, with alternatives such as a scale. For the calculation of reliability, the Cronbach alpha method was used, where a value of 0.84 was obtained, determining that the instrument was highly reliable. The data obtained were tabulated according to the reagents and interpreted statistically by absolute and relative frequency, calculating the scores of central tendency and standard deviation, represented by tables. The results indicate that the applied management strategies establish the coherent sequence of the actions to be carried out, for which reason they are oriented to the development of the competences of the employees, by virtue of aligning with the needs and processes that arise from the environment to navigate in the competitiveness of these organizations. Establishing that a properly formulated strategy carries with it the scope of established objectives.
\end{abstract}

Keywords: Management strategies, competencies, competitiveness, needs, processes

\section{Introducción}

Actualmente las organizaciones, han visto la necesidad de efectuar transformaciones, suscitado por el proceso de la globalización, la creación de una cultura de servicio en sus empleados, repercutiendo favorablemente en su entorno; así como, la implementación de acciones estratégicas para lograr el éxito, en conjunto con actividades diseñadas para lograr brindarle a empleados una mayor área de competencia laboral. En ese contexto, una empresa debe tratar de llevar a cabo acciones que obtengan beneficios de las fortalezas internas, aprovechen las oportunidades externas, mitiguen las debilidades internas, para disminuir el impacto de las amenazas externas; en este proceso radica la esencia de las estrategias gerenciales. Igualmente, para sobrevivir en el torbellino de la dinámica actual.

En este sentido, el gran reto actual de la dirección y gestión de las organizaciones radica en crear herramientas útiles para promover la eficiencia en el personal, lo que les induzca a comprometerse con los objetivos organizacionales e integrarse en el proyecto de la empresa, para obtener ventajas sostenibles y duraderas en el tiempo. Es lógico suponer que las posibilidades de triunfo de la estrategia de una organización sean mucho mayores cuando esta concuerde con la estructura planteada para su aplicación. En consecuencia, conforme esta cambia con el tiempo, también debe hacerlo la estructura.

$$
\text { Bajo este contexto, Cantú (2002) }
$$
considera que las estrategias deben estar determinadas con respecto a tiempo, espacio y objetivos a cumplir; razón por la cual se deben diseñar bajo un plan, en este orden de ideas, es concebida como un proceso orientador, el cual proporciona los lineamientos para definir los objetivos organizacionales, los recursos a utilizar, así como las políticas que orientaran la administración de dichos recursos. Asimismo, al ser aplicadas al personal influirán en su conducta dentro de la organización y por ende en su rendimiento laboral.

Sin embargo lo más importante en este

García Guiliany, Jesús E.; Durán, Sonia E.; Hernández, Janeth C. \& Moreno, María E. 
concepto es que la perspectiva es compartida, pues la estrategia es una perspectiva compartida por los miembros de una organización, así como entre ellos a través de sus intenciones y acciones. Cuando se involucra en el contexto, se entra en el ámbito de la mente colectiva; individuos unidos por afinidades de pensamiento o de comportamiento o ambos.

Aunado a ello, Mintzberg y Quinn (2003) definen la estrategia como el patrón o plan que integra las principales metas y políticas de una organización, y a la vez establece, la secuencia coherente de las acciones a realizar. También plantean que este es un modelo, específicamente, un patrón en un flujo de acciones.

La aplicación y ejecución de estrategias en las organizaciones les permite ajustarse a las necesidades del entorno, generando procesos competitivos, amplios, pero además adecuarlos a las posibilidades internas de cada empresa, para ello es necesario establecer lineamientos de acción gerencial, asumiendo retos innovadores. Para Duran, Crissien, Virviesca y Garcia (2017), las estrategias gerenciales constituye un proceso que permite a una organización ser proactiva en vez de reactiva en la formulación de su futuro. Es lógico suponer que las posibilidades de triunfo de la estrategia de una organización sean mucho mayores cuando esta concuerde con la estructura planteada para su aplicación, en consecuencia, conforme esta cambia con el tiempo, también debe hacerlo la estructura.

Lo más importante en este concepto es que la perspectiva es compartida, pues la estrategia es unaperspectiva compartida por los miembros de una organización, así como entre ellos a través de sus intenciones y acciones. Cuando se involucra en el contexto, se entra en el ámbito de la mente colectiva; individuos unidos por afinidades de pensamiento o de comportamiento o ambos.

Antes estas consideraciones, en las empresas constructoras las estrategias gerenciales se hacen relevantes por cuanto logran alcanzar los objetivos y metas en el término de pertinencias en la optimización de la eficiencia en el desempeño de las funciones y actividades de los trabajadores.

Razón por la cual, es relevante fijar los objetivos y metas en las empresas constructoras, estos representan los resultados específicos a largo plazo que la organización busca lograr; asimismo, constituyen los componentes importantes del proceso de las estrategias gerenciales, por ello el notable significado para la formación de equipos de trabajo, producto de los alcances previstos en la ejecución de las mismas. De acuerdo a lo planteado, surgió la necesidad de Identificar las estrategias gerenciales en el desarrollo de competencias laborales para crear ventaja competitiva en pymes de barranquilla atlántico.

\section{Antecedentes científicos}

Michael Porter vio en la obtención de una ventaja competitiva duradera la clave del éxito en el mercado. Es esa ventaja la que permite conseguir unas rentabilidades mejores a través de una posición favorable en su sector. Para lograrla, distinguió res tipos de estrategias genéricas 1: el liderazgo en costes, la diferenciación y el enfoque. La primera intenta obtener una posición segura consiguiendo unos costes bajos que protejan 
la posición de la empresa. La segunda intenta ofrecer productos diferenciados y la tercera intenta ofrecer un producto a la vez diferenciado $\mathrm{y}$ de bajo coste al concentrarse en un nicho.

Esta distinción de las estrategias se realizó en un momento en que las empresas perseguían mercados masivos. En la época actual, en la que los mercados se hallan altamente segmentados, la triple distinción sigue siendo válida aunque resulta un tanto burda. Frente a esta distinción de las estrategias encontramos el esquema de Treacy y Wiersema1. Estos autores dicen que las empresas deben seleccionar entre lo que llaman tres tipos de disciplinas de creación de valor $y$, entonces cultivar las capacidades que las generan. Unas empresas se centran en buscar la excelencia operacional.

Ofrecen productos de una calidad razonable a un precio bajo. El énfasis de estas empresas está en la eficiencia, en mejorar sus operaciones, logística y procesos de negocio. Otras empresas optan por el liderazgo en producto. Ofrecen productos diferenciados de prestaciones avanzadas. Para ello enfatizan la gestión de la innovación, el desarrollo de productos y la marca. La diferencia con el modelo de Porter se encuentra con la tercera disciplina, la proximidad con el cliente. Las empresas que optan por esta sobresalen en el servicio al cliente.

Conocen muy bien las preferencias de sus diferentes clientes y adaptan sus productos y servicios a sus necesidades concretas. Para ello destacan en los CRMs, en el diseño de procesos que permitan personalizar los productos $y$ servicios, en el aseguramiento de la calidad y en una cultura de servicio notable.
El concepto de estrategia proviene de la palabra griega strategos y del verbo griego stategos (jefes del ejército), tradicionalmente utilizada en el terreno de las operaciones guerreras. En los últimos años el concepto de estrategia ha evolucionado de manera tal que ha surgido una nueva escuela de administración y una nueva forma de dirigir las organizaciones, llamada “administración estratégica. Gestada como una herramienta que permite a las organizaciones prepararse para enfrentar las situaciones que se presentan en el futuro ayudando con ello a orientar sus esfuerzos hacia metas realistas de desempeño.

Para Hax y Majluf (1996), la estrategia es la dirección intencionada del cambio para conseguir ventaja competitiva en los diferentes negocios de la empresa. Carrión (2006), quién cita a estos autores afirma que esta definición aporta dos cosas importante: relaciona la estrategia con el cambio y con la creación de ventajas competitivas. Lo anterior se interpreta como la relación entre la estrategia y la búsqueda de una ventaja competitiva. Sin embargo, no siempre una compañía logra obtener su ventaja competitiva, representando esto una delgada línea entre el éxito y el fracaso.

En este orden de ideas, Drucker (2003) sostiene que el direccionamiento estratégico incluye la suma de todas las decisiones, correctas o equivocadas, las cuales determinan el futuro de una organización, producen resultados buenos o malos. Se pueden identificar dos tipos de estrategia: las planeadas (pretendidas) y las reales (logradas), también se diferencia la estrategia de un plan estratégico.

García Guiliany, Jesús E.; Durán, Sonia E.; Hernández, Janeth C. \& Moreno, María E. 
Asimismo manifiesta que se constituye en un plan a largo plazo, cuyo objetivo es desarrollar total o parcialmente los recursos humanos y materiales de la empresa, es el resultado de una decisión ejecutiva, se relaciona con la determinación del propósito organizacional, la cual debe llevarse a cabo para una planificación eficaz.

Por otra parte Cantú (2002), asume el direccionamiento estratégico como un patrón colectivo de decisiones que actúa sobre la formulación $\mathrm{y}$ despliegue de recursos de producción. En este sentido, Mintzberg y Quinn (2005) establecen que las estrategias formales efectivas contienen tres elementos esenciales: las metas, las políticas y las principales secuencias de acción.

Por tanto, el direccionamiento estratégico, se convierte en uno de los puntos centrales de los procesos administrativos de toda organización, en la cual ha de llevar acciones integradas y sistemáticas, relacionadas en un elemento con otro.

Como parte de los avances de la moderna ciencia de la Administración, se plantea en la década de los años setenta en donde surge la necesidad de estudiar la orientación y dirección que los líderes fundadores le imprimen a las organizaciones. Ansoff, Declerck y Hayes (1988), citados por Duran et al (2017) advierten un cambio en la postura respecto a la estrategia en la empresa y lo desarrollan como un paso de la planeación estratégica a la administración estratégica.

Se trata de una corriente que piensa, se debe ir más allá de la planeación estratégica, refiriéndose a la aplicación de los planes y el proceso en el cual los directivos de las organizaciones trazan una orientación básica para su negocio y buscan la manera de implementar esta línea de acción con la finalidad de alcanzar sus objetivos.

Desde esta posición Betancourt (2005) plantea que la dirección estratégica es un concepto más general que abarca el concepto de planificación estratégica. Por su parte, Ansoff (1997) expone que el resultado final de la dirección estratégica es el potencial para el logro futuro de los objetivos de la organización y como lo plantean Johnson y Scholes (2001), el alcance de la dirección estratégica es mayor que el de cualquiera de las áreas de dirección operativa, por lo que el direccionamiento estratégico comprende una integración de la estrategia en todos los niveles de la corporación.

Bajo este contexto, el proceso de la dirección estratégica ha creado su propia dinámica con diferentes enfoques que van desde modelos de intervención sobre la realidad, con esquemas de planificación deterministas y estructurados, hasta enfoques complejos de interacción. En este sentido cobra validez, la respuesta ajustada a las necesidades de cada sector productivo y la capacidad de los directivos para tomar decisiones sobre los cambios que ocurren en su entorno, de esta manera las estrategias forman parte de un conjunto de acciones orientadas hacia un fin y actúan como respuestas necesarias para la supervivencia de la organización.

Específicamente, la esencia de la estrategia consiste en la elección de una posición valiosa y única afianzada o enraizada 
en sistemas de actividades que son muy difíciles de igualar (Porter, 1996; Hill \& Jones, 2005). La delimitación del campo se orienta a sugerir que las estrategias, inicialmente comprendidas como acciones que los gerentes toman para alcanzar metas (una o varias) de la organización, son las causantes que generan un impacto importante en el desempeño el propósito de la estrategia es generar como consecuencia una meta particular o dominante: el logro de un desempeño superior al de los competidores, el cual al ser alcanzado representa una "ventaja competitiva" para la firma que lo ha logrado.

Para Garcia et al (2017) las estrategias se conciben como las acciones estudiadas para alcanzar unos fines, teniendo en cuenta la posición competitiva de la empresa y las hipótesis o escenarios sobre la evolución futura. En este sentido, la administración organizacional se enfoca en desarrollar estrategias encaminadas a lograr el buen desempeño de cualquier empresa, concerniente a la planificación, así como la dirección y control del proceso a ejecutar. Dichas expectativas gerenciales, deben ir acorde con el ambiente competitivo, en el cual están inmersas dichas empresas, por lo cual se genera la necesidad de buscar alternativas para construir una ventaja competitiva sostenible.

Al respecto, Koontz, Weihrich y Cannice (2012) consideran que una estrategia es la determinación de la misión o propósito fundamental de una empresa, la adopción de cursos de acción y asignación de recursos para lograr fines planteados. También puede ser concebida como un plan a largo plazo, cuyo objetivo es desarrollar total o parcialmente los recursos humanos y materiales de la empresa, es el resultado de una decisión ejecutiva, se relaciona con la determinación del propósito organizacional, la cual debe llevarse a cabo para una planificación eficaz.

El tema de la estrategia en la organización, en opinión de Villasmil (2016) se basa más concretamente en su diseño dentro del proceso estratégico que consiste en tomar en cuenta las fuerzas, las debilidades, las oportunidades, amenazas, los valores y las responsabilidades inherentes a la situación. Dentro de este marco, las estrategias entonces, permiten concretar y ejecutar los proyectos estratégicos. Es decir, cómo lograr y hacer realidad cada objetivo y cada proyecto estratégico. Del mismo modo el autor enfatiza en la importancia de la estrategia para ejecutar los planes de acción.

\section{Estrategias gerenciales}

Las nuevas corrientes gerenciales, establecen la necesidad de plantear estrategias, para dar respuestas al entorno tan turbulento existente hasta ahora. En estas circunstancias aquellas empresas que consigan ser sumamente eficientes podrán sobrevivir. Aunado a ello, una estrategia, es el patrón o plan que integra las principales metas y políticas de una organización, a la vez, establece la secuencia coherente de las acciones a realizar.

Por otro lado, Moreno, Et al (2017) sostiene que una estrategia adecuadamente formulada ayuda a poner en orden, además asignar, con base tanto en sus atributos como en sus deficiencias internas, los recursos de una organización, con el fin de lograr una situación

García Guiliany, Jesús E.; Durán, Sonia E.; Hernández, Janeth C. \& Moreno, María E. 
viable, del mismo modo anticipar los posibles cambios en el entorno y las acciones imprevistas de los oponentes inteligentes. Para desarrollar la variable estrategias gerenciales es importante en primer término describir el significado de gerencia.

En este orden de ideas, Robbins y Judge (2017) considera a la gerencia como un proceso que comprende las funciones de planificación, organización, dirección y control la cual se obtiene con eficacia y eficiencia, al considerar a otras personas es decir, la gerencia trabaja, se desarrolla, utilizando para ello al personal para lograr un fin determinado. En este sentido, Dell'ordine (2007) considera que la gerencia es la responsable de dirigir las actividades que sirven a las organizaciones para el logro de sus metas, por ello, al ser bien ejecutada lleva consigo el alcance de objetivos establecidos. Por otra parte, el término gerencia se define como un proceso de organización y empleo de recursos para lograr objetivos predeterminados; por ello, el objetivo global es lograr la eficiencia máxima en sus actividades al llevar a cabo las metas asignadas, en la medida que se logre alcanzar los objetivos, se revelará el grado de eficacia de las metas trazadas.

De acuerdo a esto, el gerente organiza, supervisa el trabajo y entrega el producto final. Durante este proceso de organización, los gerentes deben estar en alerta para conservar los recursos, sostienen Garcia, Duran y Prieto (2017) que producir el máximo volumen de resultados sin pérdida de recursos ni tiempo, es importante para generar competitividad. En este sentido, Mintzberg y Quinn (2005) establecen que "las estrategias formales efectivas contienen tres elementos esenciales: las metas, las políticas y las principales secuencias de acción". Por tanto, las estrategias gerenciales, se convierten en uno de los puntos centrales de los procesos administrativos de toda organización, en la cual ha de llevar acciones integradas y sistemáticas, relacionadas en un elemento con otro.

Las estrategias gerenciales es la determinación de los objetivos básicos a largo plazo de una empresa. También son cursos de acción planificados y que se diferencian generalmente por el alcance y la magnitud de la acción a seguir, la cual debe estar dirigida hacia el cambio de condiciones futuras, para el logro de los objetivos.

En este sentido, las estrategias gerenciales formuladas por los mismos autores, comprenden planes de actividad tanto formales como informales, su definición por tanto es plenamente coincidente con el concepto de para quien la estrategia es una pauta en el flujo de decisiones, por lo tanto, para Garcia, Duran, Parra y Márceles (2017), aunque los directivos de una empresa fueran incapaces de etiquetar su propia estrategia la pauta de sus decisiones definiría su estrategia real.

En tanto, Thompson y Strickland (2005) indican, que la estrategia es un plan administrativo general e integrado que relaciona las ventajas de la organización con los retos del ambiente y que tiene por objeto garantizar que los objetivos básicos de la empresa se consiguen mediante la planificación apropiada por parte de los gerentes. 
Serna (2006) define las estrategias gerenciales, como un marco de acciones que deben realizarse para mantener y soportar el logro de los objetivos de la organización, de cada unidad de trabajo, de esta manera hacer realidad los resultados esperados al definir los proyectos estratégicos.

Considerando que los autores arriba indicados coinciden en mucha de las variables del término estrategia, se acota que las estrategias gerenciales son líneas maestras de acciones unificadas comprensibles e integrales diseñado para asegurar que los objetivos básicos de la empresa sean alcanzados, en este caso en el contexto de la formación de equipos de trabajo en las empresas.

\section{Competencias laborales}

En la actualidad, los gerentes de la empresas tienen especial interés en las actitudes de sus empleados, pero además en las habilidades competencias y destrezas, ya que estos representan la fuerza productiva de la organización, de ellos depende el desarrollo de las actividades aunado al logro de los objetivos planteados, dentro de este marco se desarrolla la variable competencia laboral.

Al respecto Alles (2016) la define como las habilidades o destreza que debe tener un empleado en su ámbito laboral, también es el conjunto de atributos personales visibles que aportan al trabajo, o comportamiento para lograr un desempeño idóneo y eficiente. Las competencias laborales, integran elementos característicos del ser humano: conocimientos, habilidades y destrezas, auto concepto, rasgos y temperamento, motivos y necesidades.
Por su lado Vela (2004) define la competencia laboral como el conjunto de conocimientos (saber), habilidades (saber hacer) $\mathrm{y}$ actitudes (saber estar y querer hacer) que, aplicados en el desempeño de una determinada responsabilidad o aportación profesional, aseguran su buen logro. Asimismo, Sandoval y Pernalete (2014) señalan que la competencia laboral es la capacidad de desempeñar efectivamente una actividad de trabajo movilizando los conocimientos, habilidades, destrezas y comprensión necesarios para lograr los objetivos que tal actividad supone.

El trabajo competente incluye la movilización de atributos del trabajador como base para facilitar su capacidad para solucionar situaciones contingentes y problemas que surjan durante el ejercicio del trabajo. Ademas, Para Duran, Prieto y Garcia (2017) las características personales son la causa de un rendimiento eficiente en el trabajo, pudiendo tratarse de razones, enfoques de pensamiento, habilidades o del conjunto de los conocimientos que se aplican. Las competencias, por tanto, más que hacer una referencia a lo que una persona hace habitualmente en cualquier situación, centran su objetivo en lo que una persona sería capaz de hacer cuando se le demanda.

En síntesis, los autores coinciden en que las competencias laborales puntualizan y especifican las formas de la referencia de cualquier característica individual que pueda medirse con fiabilidad y que permita pronosticar el desempeño excelente de una persona en un puesto de trabajo.

Las competencias son comportamientos

García Guiliany, Jesús E.; Durán, Sonia E.; Hernández, Janeth C. \& Moreno, María E. 
sostenibles en la práctica laboral que permiten a una persona actuar eficazmente Benavides (2002) su aparición y permanencia se apoya en el conocimiento, el deseo y la habilidad de lograr sus objetivos, siguiendo a Benavides (2002), esta expresa que los estudios organizacionales se proyectan alrededor de tres clases de competencias, implicando el uso o discriminación dependiendo con los objetivos planteados por la organización. Estas son competencias genéricas, competencias laborales, y competencias básicas.

Según Benavides (2002), las competencias genéricas, son aquellas requeridas por una empresa, entidad, consorcio, que tienen como finalidad fortaleces la identidad ya que estas nacen de la política y los objetivos de las organizaciones. Así las competencias genéricas se establecen:

Para desempeñar satisfactoriamente un empleo, Para un grupo de empleos, implicando clasificación y estandarización por niveles. Para ingresar o permanecer de una empresa consorcio o sector.

Algunas compañías determinan una serie de competencias genéricas para todos sus empleados, generalizando de esta manera, comportamientos que son considerados esenciales para el cumplimiento de su misión o para la aplicación de sus políticas organizacionales. Para señalar clasificaciones determinadas para ámbitos específicos, por ejemplo: para la alta gerencia y para las gerencias intermedias, las cuales varían según los analistas de las distintas empresas.

El estudio de las competencias involucra inequívocamente al recurso humano, el cual es el eje principal de los mismos y cuyo conocimiento siempre es importante establecer ello va a depender de las características, además de las realidades de cada organización. Según Leboyer (2006) las competencias deben cumplir con las siguientes características:
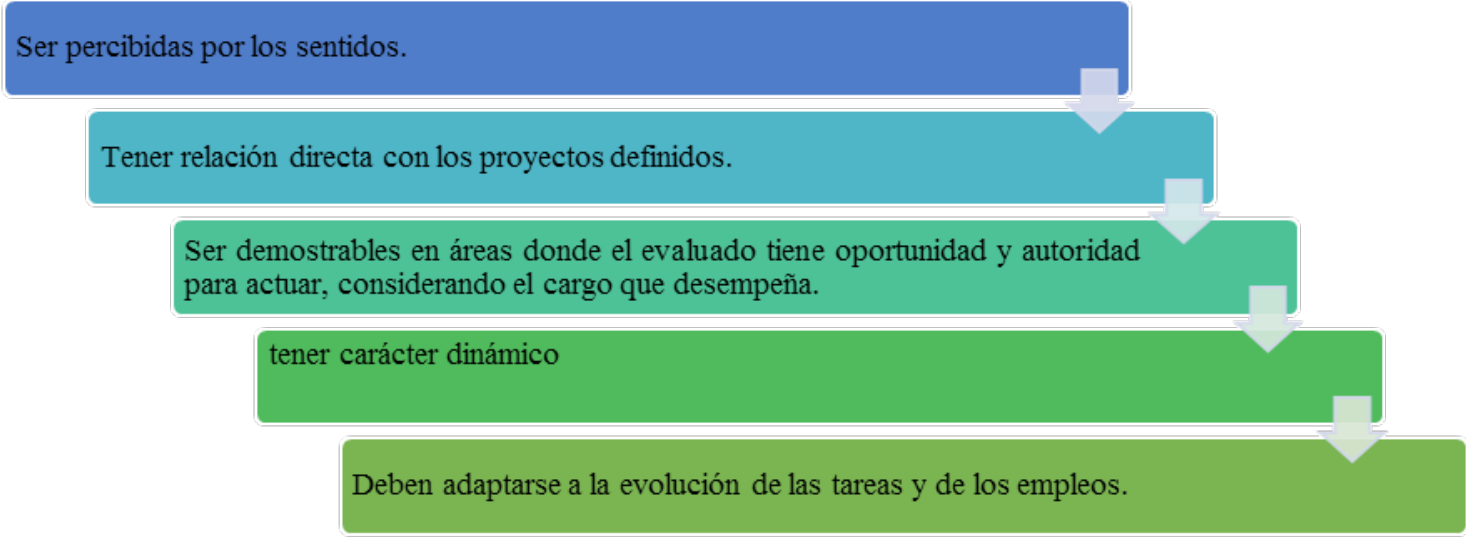

Figura 1. Características de las competencias. Leboyer (2006).

Por tanto para que las competencias de sentido el talento humano, está concebido como los empleados de una organización puedan ser el potencial a través del cual se logra la eficiencia aceptadas como tal, deben definir la actuación del y eficacia de la organización, por ende en los individuo de forma lógica y aceptable. En este institutos tecnológicos se les debe proporcionar 
los métodos para optimizar su utilización, ello permitirá el aprovechamiento de sus capacidades, por lo tanto de sus potencialidades.

\section{Metodología}

El proceso metodológico aplicado, fue de tipo descriptivo, con un diseño no experimental de campo, de corte transversal. Para obtener los datos se acudió a una población conformada por treinta nueve (39) empleados administrativos de nivel supervisorio de empresas constructoras, ubicadas en el distrito Barranquilla. Para recolectar los datos se utilizó un cuestionario constituido de 67 items, con alternativas tipo escala likert, dicho instrumento, se orientó identificar los tipos estrategias gerenciales, la eficacia de la aplicación de las estrategias, y las competencias que se promueven. Para el cálculo de la confiabilidad se utilizó el método de alfa Crombach, donde se obtuvo un valor de 0.84, determinando que el instrumento era altamente confiable.
El análisis de datos, se llevó a cabo bajo estadísticas descriptivas, tabulados de acuerdo a los reactivos e interpretados estadísticamente mediante frecuencia absoluta y relativa, calculando los puntajes de tendencia central y desviación estándar, representados por tablas.

\section{Discusión de resultados}

El proceso de aplicación de las estrategias gerenciales en las empresas constructoras. El mismo considera una dimensión y dos subdimesiones, la primera denominadaplan de acción, constituida por indicadores, el primero referido a la capacitación de empleado, este tuvo tendencias de aceptación por la mayoría de la población representado por $85,4 \%$ en función de la realización de tareas de forma eficiente, por parte del empleado, el personal al poseer más habilidades, muestra ser eficiente, además si las personas con mayores habilidades, aprenden las tareas complejas más rápido.

\section{Tabla 1}

Proceso de aplicación de las Estrategias Gerenciales

\begin{tabular}{|c|c|c|c|c|c|c|c|c|c|c|c|c|c|c|}
\hline \multirow[t]{3}{*}{ SUBDIMENSION } & \multirow{3}{*}{$\begin{array}{l}7 \\
0 \\
0 \\
\vdots \\
3\end{array}$} & \multirow{3}{*}{$\begin{array}{l}3 \\
0 \\
0 \\
>\end{array}$} & \multirow{3}{*}{$\underset{⿴ 囗 十 心}{\Theta}$} & \multirow{3}{*}{$\begin{array}{ll}N & \nabla \\
\sim & \pi \\
-1 & <\end{array}$} & \multicolumn{10}{|c|}{ ALTERNATIVAS DE RESPUESTAS } \\
\hline & & & & & \multicolumn{2}{|l|}{ TD } & \multicolumn{2}{|c|}{ DA } & \multicolumn{2}{|c|}{ NN } & \multicolumn{2}{|c|}{ ED } & \multicolumn{2}{|c|}{ TD } \\
\hline & & & & & fa & $\%$ & Fa & $\%$ & fa & $\%$ & fa & $\%$ & fa & $\%$ \\
\hline PLAN DE ACCION & 4,4 & 5 & 5 & 1,2 & & & & & & & & & & \\
\hline $\begin{array}{l}\text { Capacitación del em- } \\
\text { pleado. }\end{array}$ & 4,7 & 5 & 5 & 0,7 & 100 & 85,4 & 6 & 5,1 & 7 & 6 & 4 & 3,4 & $\mathbf{0}$ & $\mathbf{0}$ \\
\hline $\begin{array}{l}\text { Equipos autogestiona- } \\
\text { rios }\end{array}$ & 4,5 & 5 & 5 & 1 & 90 & 77 & 13 & 11,1 & 6 & 5,1 & 4 & 3,4 & 4 & 3,4 \\
\hline Pago por desempeño & 3,9 & 5 & 5 & 1,6 & 72 & 62 & 9 & 7 & 7 & 6 & 6 & 5,1 & 23 & 20 \\
\hline $\begin{array}{l}\text { EJECUCIÓN DE ES- } \\
\text { TRATEGIAS }\end{array}$ & 3,2 & 5 & 3 & 1,4 & & & & & & & & & & \\
\hline Metas & 3,2 & 3 & 3 & 1,3 & 25 & 21,3 & 25 & 21,3 & 26 & 22,2 & 25 & 21,3 & 6 & 5,1 \\
\hline Asignar los recursos & 2,5 & 1 & 3 & 1,3 & 10 & 9 & 18 & 15,3 & 37 & 32 & 10 & 9 & 42 & 36 \\
\hline Evaluar resultados & 3,8 & 5 & 4 & 1,2 & 48 & 41 & 28 & 24 & 13 & 11,1 & 26 & 22,2 & 2 & 2 \\
\hline
\end{tabular}

Fuente: Elaboración Propia

García Guiliany, Jesús E.; Durán, Sonia E.; Hernández, Janeth C. \& Moreno, María E. 
Dentro de estas perspectivas, Porter (1991), citado por Koontz (2012), sostiene que los individuos, más calificados pueden realizar tareas en forma más rápida y precisa, además este tiene mayor probabilidad de aprender tareas complejas asociadas a muchos métodos modernos de producción, que aquellos que poseen menos habilidades.

Con respecto a los equipos autogestionarios, también es parte de la estrategia de la organización, dado que el $84,1 \%$ asumieron las tendencias totalmente de acuerdo, así como de acuerdo. Referidos conocer las tareas que ejecutan sus compañeros, puede apoyar, a otro miembro del equipo, en la ejecución de sus tareas, los equipos auto dirigido, hacen las tareas más flexibles. Este resultado es apoyado por la posición de Porter (1991), citado por Koontz (2012) , quien sostiene que todos los miembros del equipo aprenden todas las tareas de este, se rotan cada una como resultado de ello, se obtiene una fuerza de trabajo más flexible y los compañeros de trabajo pueden remplazar a los compañeros ausentes.

Seguidamente el pago por desempeño, mostro una tendencia positiva, el $62 \%$ manifestó estar totalmente de acuerdo, además se le suma el $7 \%$ quienes estuvieron de acuerdo, con respecto recibir incentivos por desempeño, las bonificaciones estimula la habilidad del equipo, al recibir incentivos por desempeño, el empleado es más productivo.

Ello se sustenta con los planteamientos de Porter (1991), citado por Koontz (2012) manifiestan los autores que para nadie es un secreto que las personas trabajan por dinero, en todo caso para el investigador el pago de un incentivo por desempeño puede ayudar a incrementar la productividad del empleado. No obstante el pago de bonificaciones estimula enormemente la habilidad del equipo para satisfacer las metas de productividad.

Luego se analiza la ejecución de estrategias, incluye tres indicadores, el primero referido a las metas, los resultados muestran un comportamiento aceptable, sustentados los datos en el $42,6 \%$ de la población quienes estuvieron totalmente de acuerdo y de acuerdo. En cuanto a las metas en función del propósito a cumplir, definen el fundamento del trabajo en equipo, los equipos trabajan en forma unificada para lograr las metas.

Bajo este contexto, Gabaldón (2003), afirma que las metas en los equipos de trabajo traducen su propósito común en metas de desempeño realistas, medibles, así como específica, las metas dan la energía del equipo de trabajo, puesto que facilitan la comunicación entre sus miembros ayudan a mantenerse enfocados en obtener resultados en las empresas. Por otra parte, para el mismo autor definen el blanco final del equipo de trabajo, liderazgo estructura, proporcionan foco y dirección. Asimismo definir un enfoque común, da seguridad que el equipo de trabajo esta unificado en los medios para alcanzar sus metas.

Con respecto a la asignación de recursos, este aspecto no se cumple a cabalidad, el $44 \%$ suma las tendencias negativas en totalmente en desacuerdo, en desacuerdo. En relación a la asignación de los recursos necesarios para ejecutar las actividades laborales, los recursos 
varían en función de las actividades formuladas, la calidad de los recursos influye en el desarrollo efectivo del trabajo. Ello se contradice con lo planteado por Gabaldón (2003), quien sostiene que la asignación de recursos se orienta una vez señaladas las actividades, en ese momento se deben identificar los recursos necesarios para realizarlas.

Por otro lado David (2013) manifiesta que la implementación productiva requiera de reasignación de recursos a departamentos, fijación de normas de desempeño, instalación de sistemas de información, fijación de sistemas de recompensa, cambios en la estructura de una organización, creación de nuevos territorios de ventas, adiestramiento de nuevos gerentes y empleados, motivación de personal, obtención de nuevo capital, desarrollo de nuevas estrategias publicitarias, desarrollo de presupuestos y programas.

En este orden de ideas, se presenta el indicador evaluar resultados, sus tendencias son de que se lleva a cabo, sustentando en el $41 \%$ de la población considero estar totalmente de acuerdo, un $24 \%$ de acuerdo, en referencia a evaluar la metodología utilizada para ejecutar el trabajo, ejecutan acciones estructuradas para evaluar los resultados de las estrategias, asimismo la evaluación de los resultados es multidireccional.

En este orden de ideas, Castillo y Cabrerizo (2006), señalan que la evaluación es un elemento significativo de la acción, afecta no solo de constatar su aplicación, desarrollo y resultados, con el fin de mejorarlos, los procesos organizacionales son en principio, siempre perfectibles. Pero además este proceso involucra un diseño abierto y dinámico, el cual conlleva un conjunto de acciones adecuadamente estructuradas, a lo largo del tiempo con una secuencia determinada de intenciones formativa, asimismo medir los resultados del proceso organizacional.

\section{Tabla 2}

\section{Competencias actuales del personal}

\begin{tabular}{|c|c|c|c|c|c|c|c|c|c|c|c|c|c|c|}
\hline \multirow{3}{*}{ INDICADORES } & \multirow{3}{*}{$\begin{array}{l}\overrightarrow{0} \\
0 \\
3 \\
3\end{array}$} & \multirow{3}{*}{$\begin{array}{l}3 \\
0 \\
0 \\
>\end{array}$} & \multirow{3}{*}{$\begin{array}{l}3 \\
\stackrel{3}{\theta} \\
\underline{-1}\end{array}$} & \multirow{3}{*}{ 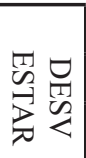 } & \multicolumn{10}{|c|}{ ALTERNATIVAS DE RESPUESTAS } \\
\hline & & & & & \multicolumn{2}{|c|}{ TD } & \multicolumn{2}{|c|}{ DA } & \multicolumn{2}{|c|}{$\mathbf{N N}$} & \multicolumn{2}{|c|}{ ED } & \multicolumn{2}{|c|}{ TD } \\
\hline & & & & & fa & $\%$ & fa & $\%$ & fa & $\%$ & fa & $\%$ & fa & $\%$ \\
\hline Competencias de logro y acción & 2,8 & 4 & 3 & 1,26 & 8 & 7 & 33 & 28,2 & 22 & 19 & 29 & 25 & 25 & 21,3 \\
\hline Competencias gerenciales & 3,1 & 3 & 3 & 1,32 & 22 & 19 & 22 & 19 & 38 & 32,4 & 16 & 14 & 19 & 16,2 \\
\hline Competencias cognoscitivas & 3,1 & 4 & 3 & 1,36 & 20 & 19 & 29 & 25 & 30 & 26 & 16 & 14 & 22 & 19 \\
\hline Competencias de eficacia personal & 3,2 & 4 & 4 & 1,44 & 25 & 21,3 & 32 & 27,3 & 18 & 15,3 & 18 & 15,3 & 24 & 21 \\
\hline
\end{tabular}

Fuente: Elaboración Propia

Las competencias actuales del personal, que favorezcan la formación de equipos de trabajo en las empresas constructoras. $\mathrm{Su}$ dimensión competencias actuales del personal, se integró por cuatro indicadores, en cuanto a las competencias de logro y acción, sus resultados generaron tendencias negativas, un 25\% estuvo en desacuerdo, además el $21,3 \%$ totalmente en desacuerdo, ello permite afirmar que en las empresas constructoras el empleado no siente

García Guiliany, Jesús E.; Durán, Sonia E.; Hernández, Janeth C. \& Moreno, María E. 
preocupación por el orden delárea de trabajo, tiene poca iniciativa para la búsqueda de información, por otra parte no tienen la capacidad de actuar para establecer relaciones cordiales.

En contraposición, se cita Alles (2016), ella define estas actitudes como la capacidad para actuar con velocidad y sentido de urgencia cuando se deben tomar decisiones importantes para cumplir con los competidores o superarlos, atender a las necesidades del cliente o mejorar a la organización. Así mismo expone que la iniciativa hace referencia a la actitud permanente de adelantarse a los demás en su accionar. Es la predisposición a actuar en forma proactiva. Implica marcar el rumbo a través de acciones concretas. Con respecto al orden, la claridad, precisión: es la preocupación constante por controlar el trabajo y la información. Implicando la insistencia en la claridad de la responsabilidad, de funciones asignadas.

En el mismo orden de ideas, están las competencias gerenciales, para ella los resultados fueron $19 \%$ totalmente de acuerdo, así como otro 19\% de acuerdo, se demuestra que en estas empresa hay capacidad para comprender las relaciones de poder, se promueve la habilidad para participar activamente de una meta común, así como la habilidad para orientar la acción de los grupos humanos. Al respecto Alles (2016) sugiere que las competencias gerenciales contienen al desarrollo de personas; dirección de personas, trabajo en equipo y cooperación liderazgo. Ello permite a la organización adecuarse a las necesidades del equipo, pero a su vez de los objetivos a cumplir.

Seguidamente se presentan las competencias cognoscitivas el $27 \%$ de la población asumió estar de acuerdo, el 17\% totalmente de acuerdo evidenciándose la capacidad para realizar un análisis lógico de los procesos, el uso del razonamiento conceptual en las empresas, igualmente el personal posee la experiencia técnica, requerida para ejecutar el trabajo. En este sentido Alles (2016) afirma que estas incluyen el pensamiento analítico, el razonamiento conceptual, así como la experiencia técnica, profesional y de dirección, por otro lado se involucra la capacidad que tiene una persona para realizar un análisis lógico en general.

Finalmente el indicador competencias de eficacia personal, sus resultados se estimaron de la siguiente manera, el $27,3 \%$ de acuerdo, $21,3 \%$ totalmente de acuerdo, ello indica que en las empresas constructoras existe, capacidad para realizar un análisis lógico de los procesos de trabajo, capacidad para desarrollar con éxito las tareas, aplicación de diversos enfoques para resolver un problema.

Sustentando estos resultados en la posición de Alles (2016), ella plantea que esta comprende el autocontrol; confianza en sí mismo; comportamiento ante los fracasos y flexibilidad La confianza en sí mismo, en relación al convencimiento de que se posee la capacidad para desarrollar con éxito una tarea o elegir un enfoque adecuado para resolver un problema.

\section{Conclusiones}

El proceso de aplicación de las estrategias gerenciales en las empresas constructoras, refiere que el personal posee habilidades que le permiten mostrar sus capacidades, por ende ser eficiente, 
además esto les permite aprender las tareas complejas más rápido. Además como parte de la estrategia de la organización, están los equipos autogestionarios, la mayoría del personal conoce las tareas ejecutadas por sus compañeros, se constató la existencia del apoyo a los miembros del equipo, de esta manera las tareas se han hecho más flexibles. En el mismo orden de ideas, los empleados reciben incentivos por desempeño, afirmando que esto estimula la habilidad del equipo, por ende son productivos.

En cuanto a las metas en función del propósito a cumplir, definen el fundamento del trabajo en equipo trabajan en forma unificada para lograr las metas, luego en la asignación de los recursos necesarios para ejecutar las actividades laborales, los recursos varían en función de las actividades formuladas, la calidad de los recursos influye en el desarrollo efectivo del trabajo. En este sentido también se hacen evaluación de resultados en forma objetiva, ejecutando acciones estructuradas para evaluar los resultados de las estrategias

Las competencias actuales del personal, que favorezcan la formación de equipos de trabajo en las empresas constructoras. Se concluye que en las empresas constructoras el empleado no siente preocupación por el orden del área de trabajo, tiene poca iniciativa para la búsqueda de información, no tienen la capacidad de actuar para establecer relaciones cordiales. En cuanto a las competencias gerenciales, se demuestra que en estas empresas hay capacidad para comprender las relaciones de poder, se promueve la habilidad para participar activamente de una meta común, así como la habilidad para orientar la acción de los grupos humanos.

Con respecto a las competencias se manifestó cierta capacidad para realizar un análisis lógico de los procesos, el personal posee la experiencia técnica, requerida para ejecutar el trabajo. Asimismo en las competencias de eficacia personal, en las empresas constructoras existe, capacidad para realizar un análisis lógico de los procesos de trabajo, capacidad para desarrollar con éxito las tareas, aplicación de diversos enfoques para resolver un problema.

\section{Referencias bibliográficas}

Alles, M. (2016). Gestión de personas. Buenos Aires: Editorial Granica.

Benavides, O. (2002). Competencias y competitividad: Diseño para organizaciones latinoamericanas. Colombia: McGraw- Hill.

Castillo, S., \& Cabreizo, J. (2003). Evaluación de programas de intervención socioeducativos ámbitos y agentes, Madrid: Editorial Pearson.

David, F. (2013). Conceptos de administración estratégica. México: Editorial Pearson, décimo cuarta edición.

Dell'ordine, J. (2007). Estrategias aplicadas al aprendizaje. Revista en Ciencias Empresariales y Ambientales, $\mathrm{N}^{\mathrm{o}}$ 2, 2007.

Durán, S. (2017). Procesos Multiculturales. Organizaciones exitosas. Bogotá, Colombia: Editorial Educoe.

Durán, S., García, J., Crissien, J., \& Virviesca, J. (2017). Estrategias gerenciales para

García Guiliany, Jesús E.; Durán, Sonia E.; Hernández, Janeth C. \& Moreno, María E. 
la formación de equipos de trabajo en empresas constructoras del Caribe Colombiano. Revista Espacios, 38(13), 26-28.

Gabaldón, F. (2003). Gerencia de Organizaciones de Servicio. Venezuela: Editorial Minerva C.A.

García, J., Durán, S., Cardeño, E., Prieto, R., García, E., \& Paz, A. (2017). Proceso de planificación estratégica: Etapas ejecutadas en pequeñas y medianas empresas paraoptimizarlacompetitividad. Revista Espacios, Volumen 38 numero 52, p. 16-32.

García, J., Durán, S., Parra, M., \& Márceles, V. (2017). Dirección estratégica del talento humano para el fomento de valores en los cuerpos policiales venezolanos. Espacios, 38(32), 16-28. En http://www.revistaespacios.com/ a17v38n32/a17v38n32p16.pdf.

Garcia J., Durán, S., \&, Prieto R. (2017). Políticas de gestion de talento humano para el desarrollo de competencias gerenciales en empresas metalmecánica. FACE: Revista de la Facultad de Ciencias Económicas y Empresariales, 17(2), 130-141.

Hill, Ch. \& Jones, G., (2007). Administración estratégica 5ta edición. Colombia: Editorial Mc graw Hill.

Levy-Leboyer, C. (2000). Gestión de las competencias: cómo analizarlas, como evaluarlas y como desarrollarlas. Barcelona: Gestión.
Mintzberg, E. \& Quinn, B. (2005). El proceso estratégico. Conceptos, contextos y casos. México: Prentice hall hispanoamericana, S.A.

Moreno, Z., Parra, M., Villasmil, M., Hernández, B., \& Durán, S. (2017). Importancia del Pensamiento Estratégico y Acciones Estratégicas para impulsar el emprendimiento social en las universidades Venezolanas. Revista Espacios. Volumen 38, Número 45. p.4 En: http:/www.revistaespacios.com/ a17v38n45/17384504.html

Robbins y Judge, S., \& Judge, T. (2017). Comportamiento organizacional. México: Editorial Pearson., Decimoséptima edición.

Sandoval, F., \& Pernalete, D. (2014). Marco de trabajo para gestionar las competencias laborales.Enl@ce RevistaVenezolanade Información, Tecnología y Conocimiento, $11(3), 11-32$

Serna, H. (2006). Gerencia estratégica: Planeación y gestión - teoría y Metodología, incluye guía para el diagnóstico estratégico, Colombia, 3R editores.

Thompson, A. \& Strickland, A. (2005). administración estratégica 11va edición. México: Editorial McGraw- Hill.

Vela, L. (2004). Gestión por competencias: el reto compartido del crecimiento personal y de la organización. Esic Editorial. 